\title{
Sensitivity analysis for a proposed sewer overflow screening device
}

\author{
$\underline{\text { M. A. Aziz }}$, M. A. Imteaz ${ }^{a}$ and M. Samsuzzoha ${ }^{a}$ \\ ${ }^{a}$ Faculty of Engineering \& Industrial Sciences, Swinburne University of Technology, Melbourne, Australia \\ Email: aaziz@swin.edu.au
}

\begin{abstract}
Sewer overflows to receiving water bodies cause serious concerns for the environment, aesthetics and public health. To overcome these problems a self-cleansing, low maintenance, high capture efficiency and less expensive device was developed and tested at Swinburne University of Technology, Melbourne. There are a number of different screening systems used in sewer overflow screening devices. Most of the screener has the common drawbacks in the available commercial devices include inadequate screening capacity, external power needs and high cost. To overcome such drawbacks a new overflow sewer device, known as the 'Comb Separator' was proposed. The device has no moving parts, a robust stop/start operation, an effective self-cleansing mechanism, low maintenance and operation costs and no external power requirements.
\end{abstract}

The proposed experimental device in the present research is sewer gross pollutant trapping device, which consists of a rectangular tank and a sharp crested weir. In front of the weir a series of vertical, parallel combs to separate entrained sewer solids from the overflow as shown in Figure 1. The studied device was tested with a series of sewer solid materials including condoms, tampons, cigarette butts, cotton buds, bottle caps, wrap papers etc. Larger sewer particles (greater than $10 \mathrm{~mm}$ diameter) can be captured relatively easily with capture efficiency more than $90 \%$. This capture efficiency was tested with different input varying condition; however the output capture efficiency has insignificant variation from varying input parameters. However, significant variation observed from varying input parameters for smaller particles. Hence focus of this work is to parameter sensitivity on smaller sewer solid particles.

To improve output capture efficiency of these smaller particles, important input parameters like flow conditions, layers of combs and spacing of combs and weir opening were changed and tested with different trials. Average capture efficiency varies from $50 \%$ to $85 \%$, however at times capture efficiency varied without varying input parameters, which triggered the need for a detail investigation of the parameter sensitivity. A total of forty (40) sets of experimental data were collected on eight different sets of experimental setup. Based on the experimental experience, four input parameters (flow volume, effective combs spacing, weir opening and number of comb layers) were identified influential on output sewer capture efficiency. Four different method of sensitivity testing were adopted these are Partial plots, Partial Correlation Coefficient (PCC), Sensitivity Index (SI) and Regression Analysis.

The partial plots suggest that inflow volume have a negative correlation with the output sewer capture efficiency. The $1^{\text {st }}$ comb spacing has a positive correlation whereas the $2^{\text {nd }}$ comb separator has a negative correlation. The weir opening has a positive correlation as wider weir area will reduce velocity and increase capture efficiency. Partial correlations suggest that weir opening is the most important parameter followed by $2^{\text {nd }}$ comb spacing, inflow volume and $1^{\text {st }}$ combs spacing. Sensitivity Index suggests all four parameters are varied 5\% only for their sensitivity index. Regression analysis did not consider weir opening and comb layers as input parameters since these parameters failed to satisfy normal distribution assumption. Effect of $1^{\text {st }}$ and $2^{\text {nd }}$ comb spacing are combined in effective comb spacing (which shows bell shape trend of the input parameters) along with inflow volume as input parameters. Results shows inflow volume has significant influence output capture efficiency of the output parameter. Effective comb spacing combine the effect of comb does not provide any particular trend over capture efficiency although $1^{\text {st }}$ comb has a positive correlation and $2^{\text {nd }}$ comb spacing has a negative correlation with the capture efficiency.

Keywords: $\quad$ Sewer Solids, Screening Device, Sensitivity Analysis, Trapping Efficiency 


\section{INTRODUCTION}

Under wet weather conditions, sewer overflows cause serious concern to the environment, aesthetics and public health concern. These sewer solids either disperses float or wash into rivers and eventually settle; creating odours and toxic/corrosive atmospheres in bottom mud deposits. It creates aesthetic upsets either in general appearance like increasing dirtiness or in the actual presence of specific, objectionable items such as float debris, sanitary discards/faecal matter, scum or even parts of car tyres. To address these problems, active research had involved different types of screening devices. Screening of sewer solids is a controlled process that is desirable to the sewer system. It needs to be automated in order to ensure operational safety and effective use in unstaffed remote locations. Most environmental regulations prefer floatable controls and safety to device failed conditions. These requirements trigger the need to research the different types of screening devices and screenings handling systems to select the most appropriate for a particular installation, especially at isolated locations. To make this device practically applicable to active sewer systems a series of sensitivity analysis needs to be performed to gain a thorough understanding of the device placed in unstaffed remote locations. Figure 1 shows the photograph of the device under testing condition.

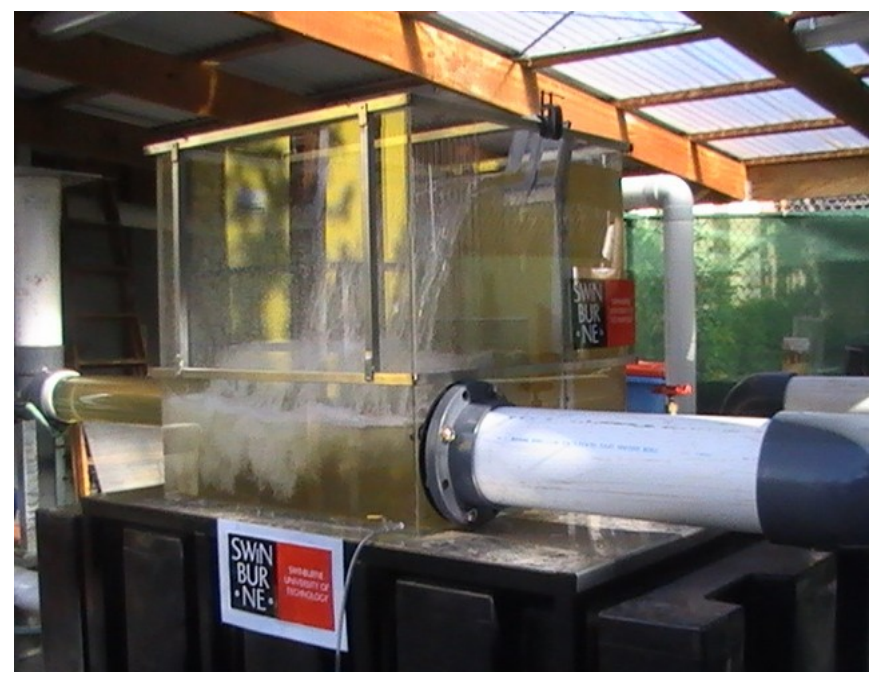

Figure 1. Experimental setup for sewer overflow device.

A 'sensitivity analysis' of such device is not only critical to ensure optimisation and validation but also to serve as a guide to future improvement opportunities of proposed experimental devices. The sensitivity analyses of the experimental data can help to understand the following:

- Which input parameter require additional research for strengthening knowledge and understanding to reduce output uncertainty

- Which input parameter can be neglected and eliminated from the final model

- Which input contributes the most to the output variability

- Which parameters are most highly correlated with the output capture efficiency

- Once the device is in practical use, what would be the consequent results from changing a given input parameter

\section{METHODOLOGY}

Some of the common drawbacks in the available commercial devices include inadequate screening capacity, external power needs and high cost (Simon et al., 2008). Faram et al. (2001) tested a hydro jet device installed in USA, Australia and mainland Europe. However, in all cases the device was directly associated with blockages of the sewerage system. To overcome such drawbacks a new overflow sewer device, known as the Comb Separator, was proposed and tested at Swinburne University (Phillips et al., 2010 and Aziz et al., 2011). The device has no moving parts, a robust stop/start operation, an effective self cleansing mechanism, low maintenance and operation costs and no external power requirements.

In the present study, the sewer overflow device consists of a rectangular tank and a sharp crested weir that are followed by a series of vertical, parallel combs to separate entrained sewer solids from the overflow. The device does not require electrical or mechanical power for the self-cleansing mechanism, enabling the device to work efficiently in unstaffed remote locations. In addition, it has no moving parts and has robust stop/start operation and low maintenance. The performance of the device is based on its sewer solids capture efficiency. A series of laboratory tests conducted at Swinburne University of Technology, Melbourne used a range of experimental conditions to simulate conditions in existing urban sewerage systems. These included 
different overflow/ spill rates, spacing of combs, layer of combs, weir openings. Forty (40) sets of experimental data were collected based on eight (8) different sets of experimental conditions. These experimental experiences provide the opportunity to understand the input output relation of the experimental device.

The experimental work was restricted by the physical limitations of conducting experiments for all different combinations of the experimental conditions. This is inherent in any laboratory studies. In addition, experimental work involves significant cost and time. To overcome such limitations a model is proposed based on the four input parameters (flow volume, effective comb spacing, weir opening, and layers of combs) and sewer capture efficiency as output parameter. Sensitivity of these parameters is of paramount importance in considering the ability of this device to function property in remote, unmanned locations. Understanding and analysing model sensitivity and uncertainty has been an active theme of research for hydraulic engineers for many years (Hall et al. 2009). Sensitivity analysis is predominantly used in design variables for hydraulic experimental parameters which will be decided upon by standard engineering practice.

\section{EXPERIMENTAL MECHANISM}

The laboratory device is connected to an inlet pump and inlet pipe. Two outlets are mounted on the device, one to convey overflow water away and the other to drain the sewer water remaining in the storage chamber. A series of combs to segregate sewer solids from the sewer overflow are mounted next to the sharp crested weir, refer to Figure $2 \mathrm{a}$.

Phase 1: After the start of precipitation overflow the storage chamber fills with sewage. A floating ball at the bottom of the sewer solids holding chamber then closes at this point as shown in Figure $2 \mathrm{a}$. As the overflow continues, the storage chamber overflows above the sharp crested weir. The captured sewer solids are intercepted by the parallel combs and fall into the holding chamber (pollutant capture chamber), (Operational procedure of the experimental condition).
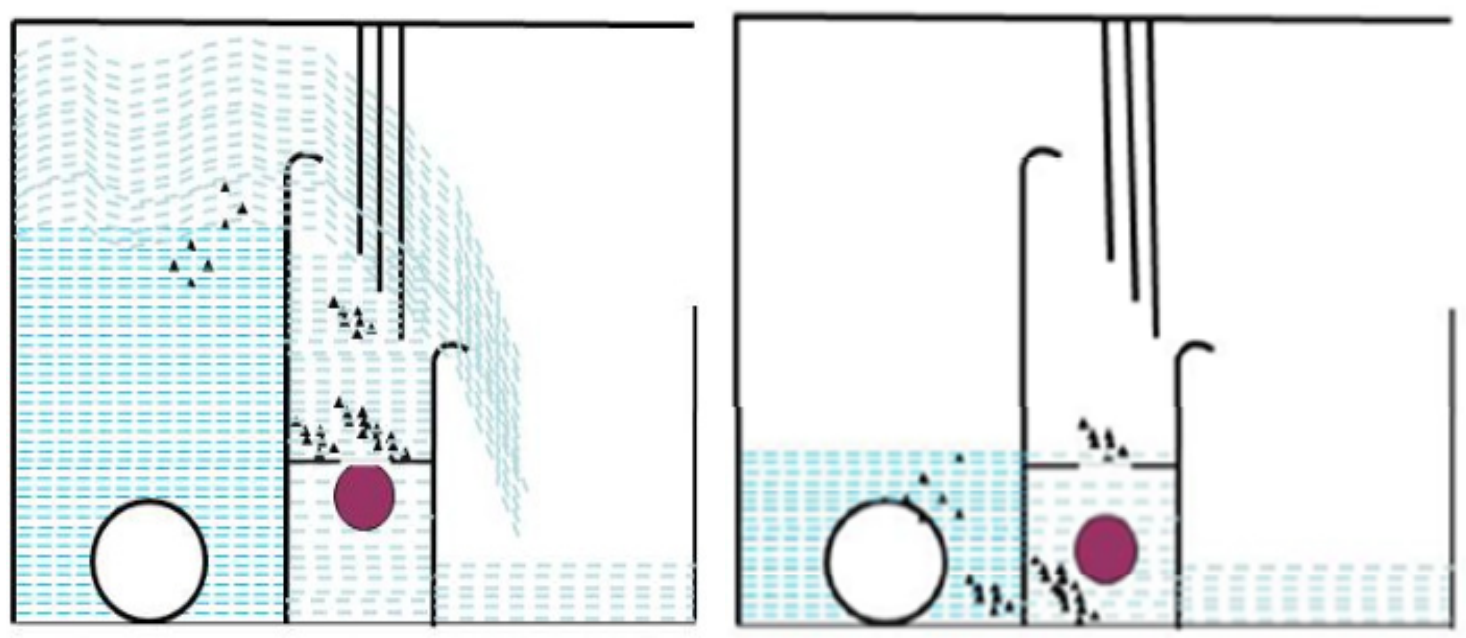

Figure 2a. Operational procedure of the new Sewer Overflow Screening Device Phase 1, Figure $2 \mathbf{b}$. Operational procedure of the new overflow screening device Phase 2

Phase 2: After cessation of precipitation, the water level within the storage chamber falls below the valve level. The low pressure of the liquid in the sewer solids holding chamber allows the ball to drop and flushes the entire captured sewer solids back into the storage chamber, refer to Figure $2 b$ (Screening mechanism, operational phase 2).

For our experiments, common sewer solids like condoms, tampons, cigarette butts, wraps, cotton balls and bottle caps were tested. The experimental conditions were varied with different flow volumes and number spacing of combs layers. The sharp crested weir was effective to handle device failed conditions and bypass to the outflow chamber.

\section{DATA ANALYSIS}

To understand the sensitivity of the experimental device an input output relation model was developed based on the learning from hydraulic experiments (Aziz et al., 2013). Four input parameters (Flow volume, 
effective comb spacing (spacing of $1^{\text {st }}$ Comb, Spacing of $2^{\text {nd }}$ Comb), Weir Opening, Layer of Combs) could have impacted the output sewer solid capture efficiency. To get the optimum experimental conditions the following eight (8) different experimental set ups were used.

Table 1. Experimental results of sewer solids less than $10 \mathrm{~mm}$ dimension.

\begin{tabular}{|c|c|c|c|c|c|c|c|}
\hline & & & \multicolumn{4}{|c|}{ Effective Comb Spacing } & \\
\hline $\begin{array}{l}\text { Experimental } \\
\text { Setup }\end{array}$ & $\begin{array}{c}\text { Flow } \\
(\mathrm{L} / \mathrm{s} / \mathrm{m})\end{array}$ & $\begin{array}{l}\text { Flow } \\
(\mathrm{L} / \mathrm{s})\end{array}$ & $\begin{array}{l}\text { Spacing of } \\
1 \text { st Comb }\end{array}$ & $\begin{array}{l}\text { Spacing of } \\
\text { 2nd Comb }\end{array}$ & $\begin{array}{c}\text { Weir } \\
\text { Opening }\end{array}$ & $\begin{array}{l}\text { Layer of } \\
\text { Combs }\end{array}$ & $\begin{array}{c}\text { Average } \\
\text { Capture } \\
\text { Efficiency } \\
(\%)\end{array}$ \\
\hline 1 & 53 & 27 & 25 & 25 & 510 & 3 & 50 \\
\hline 2 & 46 & 45 & 25 & 25 & 970 & 3 & 68 \\
\hline 3 & 46 & 45 & 20 & 20 & 970 & 3 & 68 \\
\hline 4 & 31 & 30 & 15 & 15 & 970 & 2 & 87 \\
\hline 5 & 76 & 35 & 15 & 15 & 460 & 2 & 56 \\
\hline 6 & 77 & 36 & 20 & 20 & 470 & 3 & 59 \\
\hline 7 & 72 & 34 & 12.5 & 15 & 470 & 2 & 55 \\
\hline 8 & 67 & 31 & 25 & 10 & 470 & 2 & 84 \\
\hline
\end{tabular}

Four different sensitivity analysis processes were adopted. Other than regression analysis data was completed based on Hamby (1994). The model equations for sensitivity analysis are given below.

The proposed model would be shown by the formula below:

Input Parameters (Flow volume, Effective Comb Spacing, Weir Opening and Layers of Combs) = Output Parameter (Sewer Solids Capture Efficiency)

(1)

From the experimental data, capture efficiency of the sewer solids was calculated using the formula below:

Capture Efficiency $(C . E)=\frac{\text { Total number of materials solids retained }}{\text { Total number of solids inserted with inflow }} * 100$

(2)

While adjusting the experimental set up based on capture efficiency of sewer solids we reduced the effective spacing, using $1^{\text {st }}$ and $2^{\text {nd }}$ Comb we get better trapping efficiency. So, the parameter 'effective comb spacing' was used instead of $1^{\text {st }}$ and $2^{\text {nd }}$ Comb spacing. Both input parameter 'flow volume' and 'effective comb spacing' follow normal distribution which gives the opportunity to analyse sensitivity of these parameters using regression analysis as shown below:

Capture Efficiency $(Y)=86.754-32.509 *$ Flow $(X 1)-2.479 *$ Effective comb spacing $(X 2)$

For generating the sample data, Latin hypercube sampling (LHS) technique was used using software tools like SaSAT (2008) and SPSS. Input data were processed according to Samsuzzoha et al. (2012) for each four input parameters a probability density function is defined and generates 10,000 data based on LHS considering equal-probable serial intervals. When the assumption of normality was not justified, partial plots, Sensitivity Index and correlation coefficient were used to understand the sensitivity of parameters like weir opening and comb layers.

\section{RESULT ANALYSIS AND DISCUSSION}

\subsection{Partial Plots}

The partial plots suggest that inflow volume have a negative correlation with the output sewer capture efficiency. As the flow volume increases velocity in the sewer chamber increases which force sewer solids to pass through the combs. However, as the inflow volume decreases the velocity in the chamber reduce and more sewer particles are captured in the chamber. The $1^{\text {st }}$ comb spacing has a positive correlation where as 
the $2^{\text {nd }}$ comb spacing has a negative correlation. It also reflects most sewer particles are captured in the $1^{\text {st }}$ comb separator as its spacing narrow down the rate of capture efficiency increases. By the time the sewer particles reached to the second comb it may be able to pass the comb as it generate higher velocity as passing the $1^{\text {st }}$ comb spacing. Layer of combs is insignificant with output parameter as shown in Table 1 suggest that the average of capture efficiency has a little impact on the layer of combs. The weir opening has a positive correlation as wider weir area will reduce velocity and increase capture efficiency. Partial (scatter) plots of all these parameters are shown in Figures 3 (a-d).
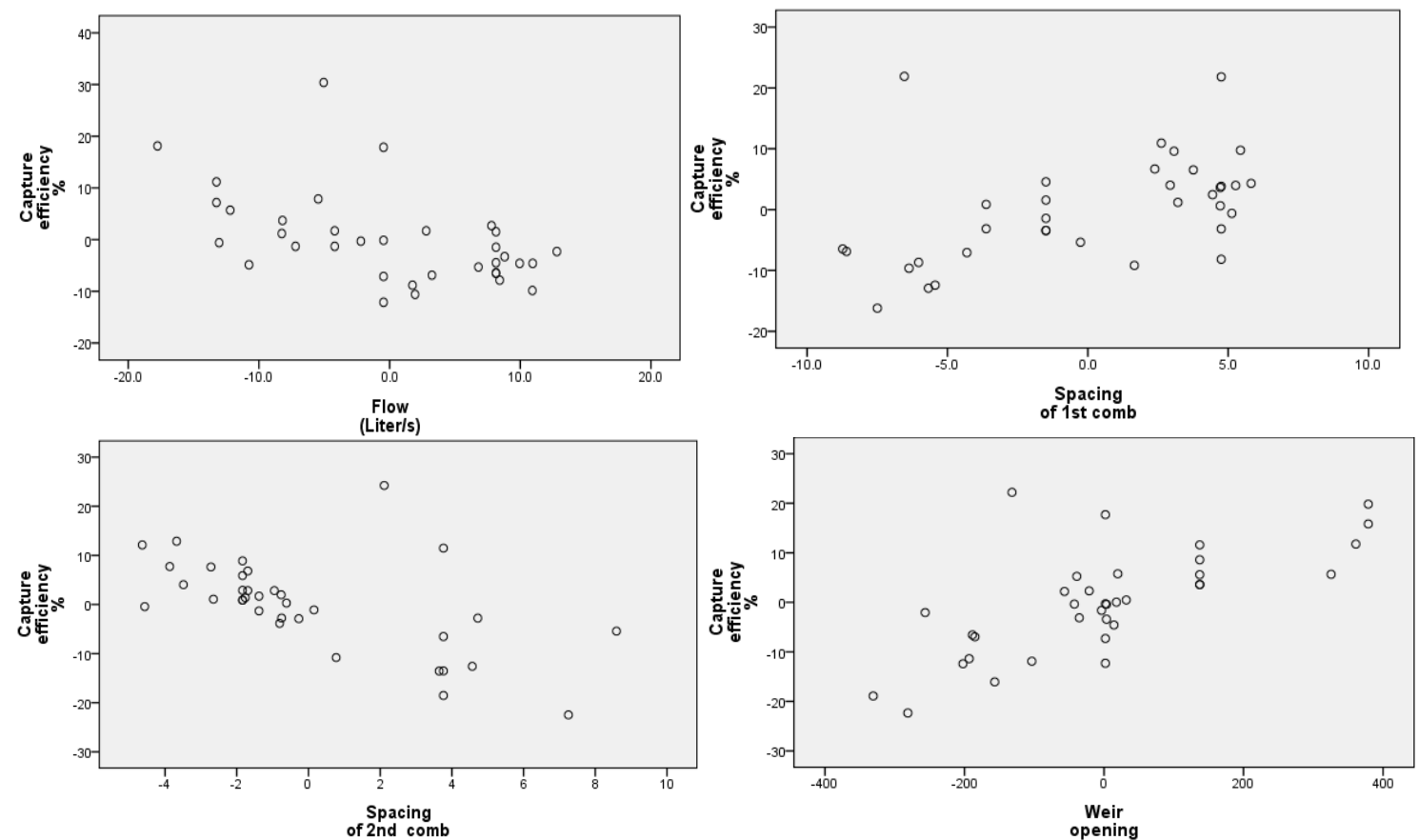

Figure 3. Partial scatter plots against output capture efficiencies for: (a) Flow, (b) $1^{\text {st }}$ Comb spacing, (c) $2^{\text {nd }}$ Comb spacing and (d) Weir opening

\subsection{Partial Correlation Coefficient}

Two predicting parameter Inflow volume and $2^{\text {nd }}$ comb spacing are significant; based on the standardized beta value the importance of the input parameter importance are Weir opening is the most important parameter, $2^{\text {nd }}$ comb spacing, inflow volume and $1^{\text {st }}$ combs spacing will follow.

\subsection{Sensitivity Index (SI)}

This is a simple approach to calculate the output \% difference when varying one input parameter from its minimum value to its maximum value (Hamby, 1994). The sensitivity result shown from this analysis is very similar for all input parameters. Sensitivity index value from this analysis found the following result inflow volume $(\mathrm{SI}=0.45), 1^{\text {st }}$ comb spacing $(\mathrm{SI}=0.5), 2^{\text {nd }}$ comb spacing $(\mathrm{SI}=0.47)$ and weir opening $(\mathrm{SI}=0.48)$. According to this analysis, $1^{\text {st }}$ comb spacing was the most significant parameter whereas inflow is the least, however the variation of these parameters are only $5 \%$.

\subsection{Regression Analysis}

Normally distributed flow volume and effective comb spacing have an R Square equal to 0.6 which expresses $60 \%$ of the output capture efficiency can be expressed by these two parameters. The adjusted R square gives us some idea of how well our model generalizes. Ideally we would like its value to be same, or very close to $(0.60-0.578=0.022$ which is $0.22 \%)$. This shrinkage means that if the model were derived from the population rather than a sample, it would account for approximately $0.22 \%$ less variance in the outcome.

The value less than 0.05 in the column Sig, the ANOVA also shows whether the model is a significant fit of the data overall. The assumption (errors in regression are independent) has been met as Durbin-Watson is 2.042 very close to 2.0 .

The histogram and scatter plots of the dependent variable, capture efficiency are shown in Figures 4 (a, b). Figures reveal that decent understanding of the normal distribution data sets were used in this model. 
Investigation on the relationships between regression standardized residual and predicted data looked like a random array of dots evenly dispersed around zero (Figure 5). This data shows no need to work on heteroscedasticity, Non-linearity or Heteroscedasticity and non-linearity from this graph output (Field, A., 2009).
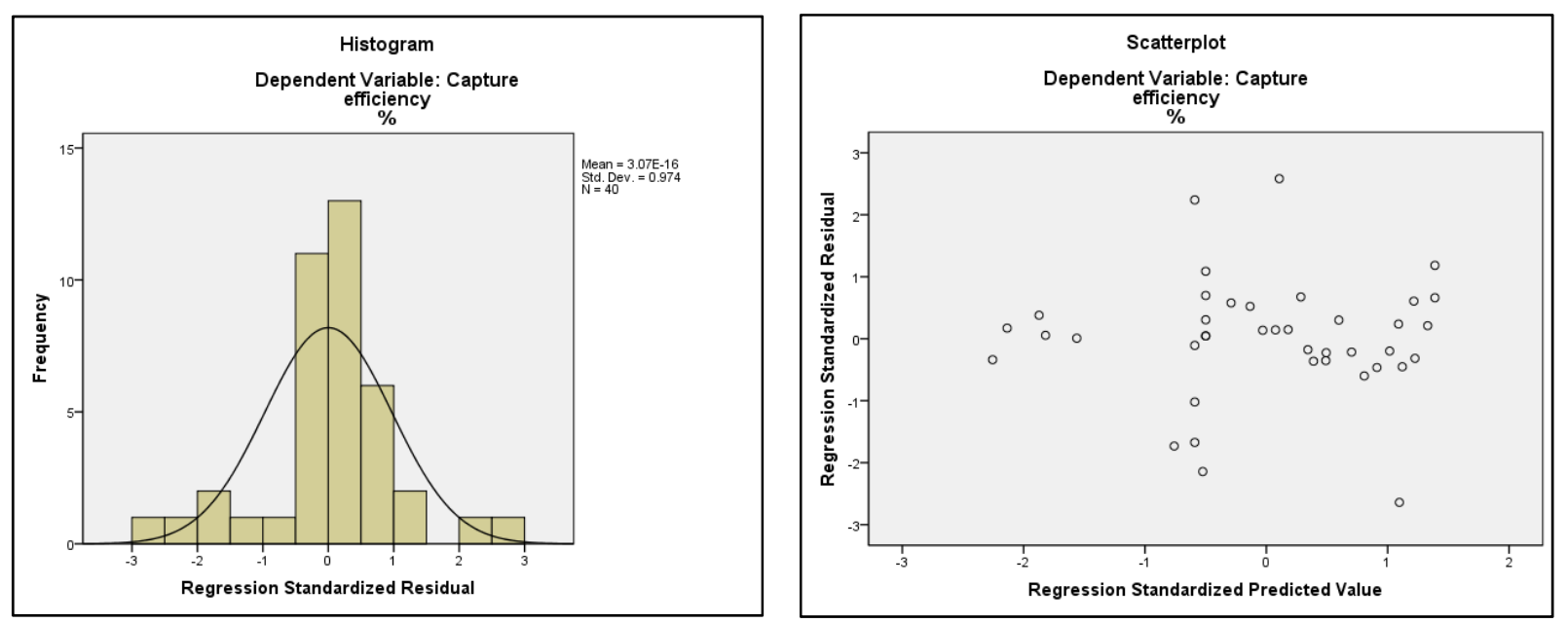

Figure 4a. Histogram plot for the proposed input output relationship. Figure 4b. Comparison of Regression Standard against predicted values

Once the mode was satisfactory to generate a reasonable input output relation, the input parameter of flow volume and effective comb spacing was tested against the overflow capture efficiency. A series of laboratory tests was carried out to evaluate the steady state. As the initial experimental device showed some initial turbulence and take up to $2 / 3$ minutes to produce a steady flow condition. Experimental run conducted varied from 6 minutes to 32 minutes with a flow conditions varies from $20 \mathrm{l} / \mathrm{s}$ to $70 \mathrm{l} / \mathrm{s}$. As the flow volume increases the capture efficiency of the sewer solids decreases. Results from 10,000 sample data shows as the flow volume increases the capture efficiency of the sewer solids decreases.
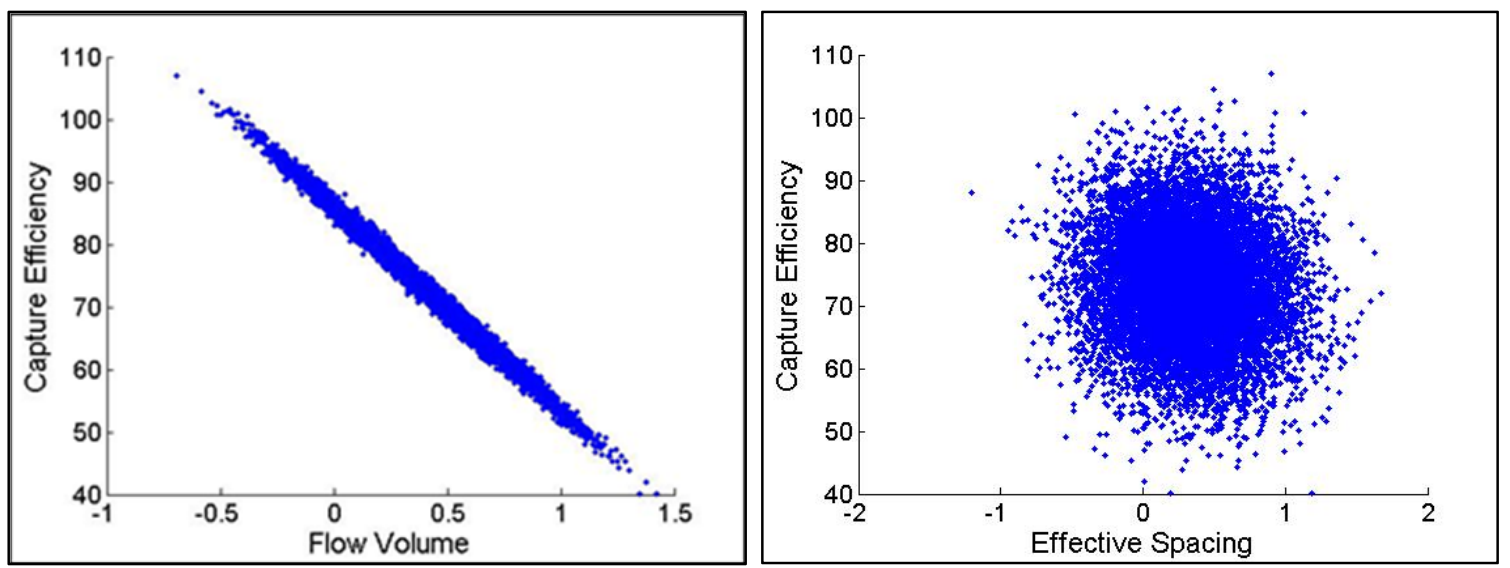

Figure 5a. Relationship between Flow Volume and Capture Efficiency. Figure 5b. Relationship between Effective Spacing and Capture Efficiency

Comb effective spacing which combined the effect of $1^{\text {st }}$ comb spacing and $2^{\text {nd }}$ comb spacing. From the capture efficiency to spacing of $1^{\text {st }}$ comb provided a positive correlation whereas with the $2^{\text {nd }}$ comb gave a negative correlation. These two effects combine in the effective comb spacing parameter which makes it difficult to follow any specific trend, refer to figure $5 \mathrm{~b}$.

\section{CONCLUSION}

A new sewer overflow device with improved capture efficiency, low maintenance, and self-cleansing mechanism was tested at the hydraulic laboratory of Swinburne University of Technology. The proposed device was free from most of the common drawbacks in the existing screening system like blinding, high maintenance requirements and electrical-mechanical switching system. It could also handle extreme events or 
device failed conditions and bypass to the outflow chamber. Most of the sewer solids of more than $10 \mathrm{~mm}$ dimension were captured with more than $90 \%$ capture rate; for materials less than $10 \mathrm{~mm}$ diameter capture efficiency varies from $50 \%$ to $80 \%$ was observed.

A series of trials with different layers of combs, spacing of combs, flow volume and weir opening were tested. Sensitivity analyses of these input parameters were performed to identify the influence of input parameters. It was found that input parameter of weir opening is the most important input parameter (has a positive correlation with output sewer capture efficiency; however as this parameter was not normality distribution additional research needed considering non-parametric testing to reduce output uncertainty. The parameter of comb layers can be neglected and eliminated from the final model based on the insignificant impact on the output results from the experimental data set, refer to table 1. Flow volume has a negative impact on the output capture efficiency, as the flow volume increases flow velocity increases and sewer capture efficiency decreases.

The effective comb spacing did not follow any particular trend against sewer capture efficiency, however $1^{\text {st }}$ comb spacing had a positive correlation and $2^{\text {nd }}$ comb spacing had a negative correlation with output capture efficiency. It also reflect importance of $1^{\text {st }}$ comb spacing as it either trap the sewer particles or help it to flow pass the second comb as it pass comb 1 due to restricted area relative velocity of the particles increases and velocity has a negative correlation with the capture efficiency.

The hydraulic experiments suggested good application potential of the proposed device in the urban drainage system. Further experiments are suggested to improve the understanding of the input parameters and provide the best practice guidelines for the device. It is also suggested that this may limit the influence on extreme events.

\section{REFERENCES}

Aziz, M.A., Imteaz, M.A., Choudhury, T.A. and Phillips, D. (2013). Applicability of artificial neural network in hydraulic experiments using a new sewer overflow screening device. Australian Journal of Water Resources, 17 (1), 77-86.

Aziz, M.A., Imteaz, M.A., Choudhury, T.A. and Phillips, D.I. (2011). Artificial Neural Networks for the prediction of the trapping efficiency of a new sewer overflow screening device. 19th International Congress on Modelling and Simulation, Perth, December 2011.

Faram, M.G., Andoh, R.Y.G. and Smith, B.P. (2001). Optimised CSO screening: A UK perspective. 4th Novatech International Conference on Innovative Technologies in Urban Drainage, Lyon, France, 25-27 June, 2001.

Field, A. (2009). Discovering Statistics Using SPSS (Third Edition). SAGE Publication Ltd., London, pp. 230-235.

Hall, J.W., Boyce, S.A., Wang, Y., Dawson, R.J, Tarantola, S. and Saltelli, A. (2009). Sensitivity Analysis for Hydraulic Models. ASCE Journal of Hydraulic Engineering, 135 (11), 959-969.

Hamby, D.M. (1994). A review of techniques for parameter sensitivity analysis of environmental models, Environmental Monitoring and Assessment, 32, 135-154.

Hoare, A., Regan, D.G. and Wilson, D.P. (2008). Sampling and sensitivity analysis tools (SaSAT) for computational modelling, Theoretical Biology and Medical Modelling, 5 (4), 1-18.

Phillips, D.I. and Simon, M. (2010). An improved method of screening sewer solids during CSO events. 7th Novatech International Conference on Urban Drainage, Lyon, France 2010.

Samsuzzoha, M., Singh, M. and Lucy, M. (2012). Uncertainty and Sensitivity analysis of the basic reproduction number of a vaccinated epidemic model of influenza. Applied Mathematical Modelling, 37 (3), 903-915

Simon. M. and Phillips, D.I. (2008). The development of a sewer solids screening system for CSO chambers. 11th International Conference on Urban Drainage, Edinburgh, Scotland, UK 2008. 